\title{
Experiencia comparada europea y el reto de la descentralización sanitaria
}

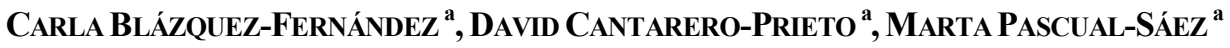 \\ a Universidad de Cantabria, Facultad CC.EE., Avenida de los Castros, s/n., 39005 Santander, \\ España.E-mail: carla.blazquez@unican.es, david.cantarero@unican.es, marta.pascual@unican.es
}

\begin{abstract}
RESUMEN
El trabajo analiza las principales características de los modelos de descentralización sanitaria en Europa prestando especial atención a los determinantes de resultados en salud y gasto. Con datos de la OECD Healht Data, se propone un modelo de panel explicativo de su comportamiento y evolución. Los resultados muestran que la renta es estática y dinámicamente el factor más importante en la explicación del volumen de gasto sanitario, mientras que otros factores de demanda y de oferta así como el grado de descentralización o tipo de sistema sanitario, son menos importantes. Mientras, en resultados en salud la descentralización tiene un efecto más dispar frente al resto de factores.
\end{abstract}

Palabras clave: Descentralización, gasto sanitario, renta, resultados en salud, Unión Europea.

\section{Comparative European Experience and the Decentralization Health Challenge}

\begin{abstract}
This paper analyzes the main characteristics of European health care decentralization models with special attention to the determinants of health outcomes and expenditures and proposes using panel data models, and data from OECD Health Data, an econometric model explaining their behaviour and evolution. The results show that income is the most important factor in explaining the volume of health expenditure both statically and dynamically, while other factors of demand and supply and the degree of decentralization or type of health system, despite also influence are less important. Instead, in health outcomes fiscal decentralization has a more mixed against other factors.
\end{abstract}

Keywords: Decentralization, Health Expenditure, Income, Health Outcomes, European Union.

Clasificación JEL: I1, H7

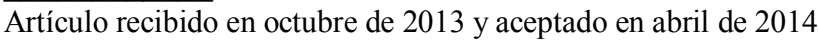

Artículo disponible en versión electrónica en la página www.revista-eea.net, ref. ə-32210 


\section{INTRODUCCIÓN}

Repensar el funcionamiento de los modelos de financiación del gasto sanitario no es una cuestión exclusiva de España. De hecho, la mayor parte de países avanzados comparten como problemática común garantizar unos sistemas sanitarios que otorguen una amplia cobertura así como altos niveles de calidad y equidad, siendo eficientes y sostenibles en el tiempo.

Resulta de interés examinar cuáles son las tendencias recientes en la financiación del gasto sanitario en otros países de nuestro entorno (Urbanos, 2004; Cabasés-Hita, 2006 y 2010; Wendt, 2009; Costa-Font, 2012). Entre ellas, aparecen los cambios en el empleo de métodos de financiación retrospectivos por los prospectivos, los introducidos en el sistema de copagos o los introducidos por los procesos de descentralización.

Así, en relación a la experiencia comparada europea y la descentralización sanitaria podemos destacar los siguientes aspectos: 1) según la OECD Health Data, la evolución demográfica muestra una clara tendencia al envejecimiento estimándose un consumo en servicios sanitarios de los mayores de 65 años superior al resto; 2) la asistencia sanitaria continua siendo uno de los sectores económicos más importantes; 3) el porcentaje de gasto sanitario sobre el PIB se ha estabilizado desde principios de los noventa. Estados Unidos sigue siendo el país del mundo con mayor gasto en sanidad; 4) en la mayoría de naciones el sector público provee la mayoría de la financiación (excepto en Estados Unidos, México y Corea), tal que el gasto público representa la mayor parte del total; 5) en la Unión Europea (UE) todos los países disponen de uno de estos dos sistemas sanitarios: i) Sistemas de Seguro Sanitario Social (SS) o ii) Sistemas Nacionales de Salud (SNS):

a) Sistemas de Seguro Sanitario Social (SS), o de corte bismarkiano (inspirados en la legislación social alemana de 1883) o sistemas profesionales en los que existe una afiliación obligatoria general donde el Estado especifica prestaciones incluidas y el suministro de servicios se reserva a fondos de aseguramiento con autonomía y autorregulación por parte de los proveedores. Se financian por cotizaciones de empresarios y empleados, a través de fondos de seguros no gubernamentales, sin ánimo de lucro, y la propiedad de los medios de producción, así como la provisión de los cuidados de salud, es pública y privada. Lo siguen países como Austria, Bélgica, Francia, Alemania, Holanda y Luxemburgo.

b) Sistemas Nacionales de Salud (SNS), que toman como base el Informe Beveridge de 1942, que formalizó la organización adoptada por Suecia en la década de 1930, cuyo fundamento es un acceso y cobertura prácticamente universal (libre acceso de todos los ciudadanos), con financiación impositiva (y esquemas de pago directo por los pacientes, en general, de escasa cuantía), una provisión directamente regulada y profesionales 
(asalariados o retribuidos a partir de fórmulas capitativas) empleados por el Estado, reconociendo para el sector privado un papel complementario o residual. Países con SNS son Dinamarca, Finlandia, Irlanda, Suecia y Reino Unido después de la II Guerra Mundial, mientras que Grecia, Italia, Portugal y España lo hicieron en los ochenta.

En este sentido es importante señalar, que el grado de descentralización sanitaria de cada país está ligado a determinantes de tipo político y al modelo empleado ("privado" o público bien de SS o SNS) en cada caso (Urbanos, 2004), siendo mayor en los modelos básicamente privados, al descentralizar éstos responsabilidades a los usuarios. De igual forma, en los SNS el grado de descentralización difiere notablemente entre países (en España las competencias sanitarias son autonómicas, Suecia delega en los condados, Italia en las regiones, en Noruega los municipios son los responsables de la atención primaria, etcétera).

Por lo tanto, con este estudio se pretende analizar las principales características de los modelos de descentralización sanitaria en Europa prestando especial atención a los determinantes de los resultados en salud y gasto, así como proponer un modelo econométrico explicativo de su comportamiento y evolución, utilizando datos de panel a fin de realizar una nueva aportación a la literatura previa.

El trabajo que aquí se presenta se organiza como sigue: en la Sección 2 se revisan los antecedentes más relevantes que encontramos en la literatura. A continuación, en la Sección 3 se señalan los ejercicios empíricos desarrollados a partir de las fuentes de datos citadas. La Sección 4 recoge los resultados. Por último, la Sección 5 se dedica a las conclusiones.

\section{DESCENTRALIZACIÓN SANITARIA: EVIDENCIA EMPÍRICA EN FACTORES DETERMINANTES DE SALUD Y GASTO SANITARIO}

\subsection{Descentralización y resultados en salud}

En los últimos años, multitud de estudios han analizado el impacto de la descentralización en diversos indicadores objetivos de salud de la población (esperanza de vida, tasa de mortalidad infantil,...). El consenso encontrado en la mayoría de la literatura especializada nos revela que existe un efecto positivo de la descentralización en dichos indicadores de salud.

Así, Mahal et al. (2000) utilizan datos de 1994 para aldeas rurales en la India para determinar si descentralización tiene influencia positiva en la mortalidad infantil, obteniéndose los coeficientes positivos esperados para los estados descentralizados. En el estudio de Robalino et al., (2001) utilizando un panel de países durante el período 1970-1995 se muestra cómo la descentralización fiscal 
(entendida como el porcentaje de los gastos del gobierno subcentral sobre el total) está inversamente relacionada con las tasas de mortalidad infantil.

Por su parte, Habibi et al. (2003) utilizan un panel de provincias argentinas para el período 1970-1994, mostrando la relación negativa existente entre descentralización de ingresos y las tasas de mortalidad infantil.

Entre los estudios más recientes encontramos el de Jiménez-Rubio, Cantarero y Pascual (2010) que utilizando un panel de 19 países de la OCDE, obtienen que la descentralización fiscal tiene un efecto sustancial y positivo en los resultados de salud. Esto corrobora los resultados mostrados en los trabajos de Cantarero y Pascual (2008) y Jiménez-Rubio (2011), para las regiones españolas y provincias canadienses respectivamente. De igual forma señalan cómo la evidencia actual sobre el impacto de la descentralización en salud es limitado porque: (i) pocos de los estudios se centran en países desarrollados, (ii) gran parte de la literatura no capta adecuadamente el nivel real de descentralización en la formulación de políticas públicas.

\subsection{Descentralización y gasto sanitario}

Existe una amplia literatura acerca de los determinantes del gasto sanitario ${ }^{1}$ $\mathrm{y}$, más explícitamente, acerca de la relación entre gasto sanitario per cápita y renta per cápita habiendo sido ésta profusamente analizada desde Kleiman (1974) y Newhouse (1977). Este tipo de literatura argumenta que existe no sólo una fuerte correlación positiva entre los gastos sanitarios y la renta, ambos en términos per cápita, de los países desarrollados, sino que la renta per cápita explica un alto porcentaje de la variación del gasto sanitario per cápita.

El debate en la Economía de la Salud (y en la econometría aplicada) acerca de la elasticidad renta del gasto sanitario se ha centrado en si la elasticidad es mayor o menor que uno ${ }^{2}$ (Bac y Le Pen, 2002) pero no ha considerado siempre al grado de descentralización del país (Kanavos y Mossialos, 1996; Di Matteo, 2003). Así, en primer lugar, la renta se configura como uno de los principales determinantes de los gastos sanitarios y la comprensión de dichos determinantes nos conduce a la siguiente cuestión: ¿cuál es la cantidad óptima de gasto sanitario en un país? (Henderson, 2002). En segundo lugar, el resultado tiene impor-

\footnotetext{
${ }^{1}$ Para una excelente revisión de la literatura sobre determinantes del gasto sanitario a nivel internacional véase Gerdtham y Jonsson (2000) y Atella y Marini (2004ayb).

${ }^{2}$ La elasticidad renta del gasto sanitario puede ser definida como el cambio porcentual en el gasto sanitario en respuesta a un cambio porcentual dado en la renta. Una elasticidad de la renta inferior a 1 clasificaría al gasto sanitario como inelástico a la renta y, por tanto, como un bien "necesario". Por otro lado, una elasticidad mayor que 1 clasificaría a la sanidad como elástica a la renta y, por tanto, como un bien de "lujo". Como es lógico, todo esto implica que si la elasticidad es mayor que 1, los gastos sanitarios se incrementarían más rápido que la renta, mientras que si es menor que 1, los gastos sanitarios se incrementarían más lentamente que la renta.
} 
tantes implicaciones de política económica en cuanto al comportamiento de los sectores público y privado y la financiación de la sanidad en el sentido de aquellos que piensan que la sanidad es una "necesidad" son más propensos a defender la intervención pública en sanidad. Por otro lado, muchos de aquellos que piensan en la sanidad como en un lujo podrían argumentar que es un bien como muchos otros y que para eso es mejor dejarlo en manos del mercado. Es decir, el viejo debate entre lo público y lo privado (Di Matteo, 2000).

Así, en primer lugar, Newhouse (1977) efectuó una regresión de los gastos sanitarios per cápita y el PIB per cápita para 13 países hacia 1970 y encontrando que "cerca del $90 \%$ de la varianza en los gastos sanitarios per cápita en esos países podía ser explicada con la variación del PIB per cápita".

Leu (1986) utilizó datos de sección cruzada para 19 países de la OCDE en 1974 y encontró elasticidades que iban de 1.18 a 1.36. Parkin et al. (1987) utilizando métodos y datos similares desde 1980 encontró elasticidades-renta de 1.12-1.18.

Brown (1987) utilizando una muestra de 20 países de la OCDE estimaba una elasticidad renta de 1.39. Gbesemete y Gerdtham (1992) estimaron el impacto de la renta per cápita en el gasto sanitario per cápita con datos de 1984 para 30 países africanos. Estos autores encontraron que la renta per cápita era el determinante más significativo de los gastos sanitarios y era aproximadamente igual a la unidad.

En Barros (1998) se tomaba como punto de partida los trabajos anteriores respecto a los determinantes del crecimiento del gasto sanitario pero en diferencias entre países en crecimiento, no en niveles. Básicamente, utilizando las mismas variables se conseguían resultados diferentes. Las características de los sistemas sanitarios que en general se pensaba que afectaban el crecimiento de los gastos sanitarios, como el envejecimiento, el tipo de sistema sanitario y la existencia de puerta de entrada al sistema no eran significativas. La única excepción era la variable renta, con una elasticidad baja pero cercana a la unidad.

Gerdtham et al. (1998) utilizando datos de panel para 22 países de la OCDE en el período 1970-1991 incluyó en su modelo variables como el número de médicos per cápita y el marco regulatorio de cada país. Entre los factores no institucionales, sólo la renta y el consumo de tabaco tenían un impacto significativo en el gasto sanitario. La elasticidad del consumo de tabaco indicaba que el gasto sanitario podría incrementarse en un $1.3 \%$ si el consumo de tabaco se incrementaba en un $10 \%$. Los países con médicos de primaria como puerta de entrada al sistema tenían menores niveles de gasto sanitario (bajando los costes al $16 \%)$. La elasticidad renta era menor que uno (0.74), en contraste con otros estudios.

Asimismo, en Sen (2005) se utilizan datos de 1990 a 1998 para 15 países de la OCDE a fin de evaluar la relación entre la renta per cápita y los gastos sanita- 
rios. Dichos datos le permiten al autor controlar por los efectos de varias covarianzas de demanda y oferta no institucionales introduciendo nuevas variables de coste u oferta. Sus resultados van en la línea de reducir las elasticidades de la renta respecto al gasto sanitario a valores entre 0.21 y 0.51 .

$\mathrm{Y}$ es que el problema de la relación entre el gasto sanitario y su principal determinante (la renta) puede también depender de la naturaleza de los datos que están siendo examinados. Así, en trabajos como los de Getzen (2001) y en Di Matteo (2003) se argumenta que la elasticidad de la renta puede variar con el nivel de análisis. Este autor encuentra que las elasticidades renta individuales son en general cercanas a cero mientras que las elasticidades renta del gasto sanitario a nivel nacional suelen ser mayores que 1 .

De cualquier forma, el grado de descentralización del sistema de salud en cada país puede ser de importancia al relacionarse con el nivel final de gasto sanitario, siendo más relevante en el caso de los gastos que en el de los ingresos, dado el carácter asimétrico de los procesos de descentralización lo que se refleja igualmente incluso en indicadores como la "estancia media" como factor de oferta o la esperanza de vida y mortalidad infantil en el caso de resultados en salud para poblaciones con cobertura en países europeos (Leu, 1986; Jiménez y Smith, 2005; Cantarero, 2006; Blazquez et al., 2013).

\section{ESTRATEGIA METODOLÓGICA}

\subsection{Fuentes de datos}

La principal base de datos utilizada en este estudio es la OECD Health Data. En concreto, tanto el gasto sanitario real y la renta per cápita por cada entidad se construirían a partir de la población de cada país deflactándola al utilizar índices de precios de consumo a ese mismo nivel (paridades de poder de compra en dólares de Estados Unidos). En este sentido, los datos relativos a gasto sanitario per cápita y renta están disponibles para un largo período de tiempo (desde 1960, hacia delante). El problema es que la información relativa a otras variables empleadas en este estudio, es por el contrario mucho más reciente, lo que limita la posibilidad de escoger períodos de estudio más amplios y justificaría en este caso que elijamos como punto de partida el año $1990^{3}$. Dichas circunstancias, dificultan a su vez, la inclusión de otro tipo de variables.

Lógicamente, puede existir alguna discrepancia en la base de datos para los diferentes países dadas las diferencias obvias en la recogida de los datos y en las definiciones de las variables empleadas. No obstante, tanto la periodicidad de la base OECD Health Data (renovable anualmente) como trabajos como los de Reinhardt, Hussey y Anderson (2002) no hacen más que sugerir que la base de

\footnotetext{
${ }^{3}$ Por lo que nuestro periodo temporal del análisis será el relativo a 1990-2010.
} 
datos de la OCDE es la más fiable y, lo que es más importante, la mejor para poder hacer comparaciones a nivel internacional.

\subsection{Metodología}

La literatura en la Economía de la Salud ha sugerido diferentes determinantes socioeconómicos, sobre los resultados en salud y el gasto sanitario per cápita. No obstante, en la investigación empírica la elección de las variables explicativas suele estar restringida por la disponibilidad y calidad de los datos ${ }^{4}$. Por lo que nuestro periodo temporal del análisis será el relativo a 1990-2010 para los quince países europeos seleccionados: Austria, Bélgica, Dinamarca, Finlandia, Francia, Alemania, Grecia, Irlanda, Italia, Luxemburgo, Países Bajos, Portugal, España, Suecia y Reino Unido.

Nuestro modelo empírico a estimar es el aplicado en los estudios de este tipo, pretendiendo a su vez, ampliarlo y adaptarlo al caso europeo. Así, la elección de las variables determinantes de los resultados en salud o del gasto sanitario, está influenciada tanto por los trabajos anteriores como por el análisis gráfico (ver ANEXO) y nuestras estimaciones preliminares. Considerando como factores determinantes los siguientes:

$$
\begin{aligned}
& S A L U D_{i t}=\beta_{0}+\beta_{1} Y_{i t}+\beta_{2} \text { OFERTA }_{i t}+\beta_{3} S_{i t}+\beta_{4} D E S C_{i t}+u_{i t} \\
& G S P C_{i t}=\beta_{0}+\beta_{1} Y_{i t}+\beta_{2} \text { DEMANDA }_{i t}+\beta_{3} \text { OFERTA }_{i t}+\beta_{4} S_{i t}+\beta_{5} D E S C_{i t}+u_{i t}
\end{aligned}
$$

- La renta $(Y)$.

- Factores de demanda (DEMANDA); entre ellos, el porcentaje de población mayor de 65 años, o la tasa de desempleo.

- Factores de oferta (OFERTA); como la media de estancias de pacientes en hospitales, el número de médicos generales (densidad por 1000 habitantes), y el número de camas (densidad por 1000 habitantes).

- Una variable dummy para el hecho de que el país tenga un sistema de Seguridad Social como indicativo de un mayor gasto sanitario.

- La descentralización sanitaria, entendida bien en términos de gasto como de ingreso, como indicativa de un mayor gasto sanitario ${ }^{5}$.

\footnotetext{
${ }^{4}$ También es cierto que muchos de los factores pueden estar fuertemente correlacionados y plantearse problemas de multicolinealidad. Puede ser ésta una de las razones por las que no todas las variables sean incluidas al mismo tiempo en el modelo econométrico que finalmente se propone.

${ }^{5}$ Un mayor grado de descentralización bien del gasto bien de ingresos relacionados con la sanidad pueden afectar positivamente al gasto sanitario total. En este sentido, señalar que medidas precisas de descentralización sanitaria son difíciles de implementar dado que dicha descentralización es un fenómeno complejo con diversos componentes políticos, fiscales y administrativos (Banting y Corbett, 2002). De todos modos, por ahora la única medida disponible de descentralización sanitaria es desde la perspectiva del gasto una medida de tipo fiscal: la ratio de gasto
} 
En este trabajo se han planteado diversos modelos de panel con el fin de capturar la heterogeneidad no observable (ya sea entre los países o en el tiempo). Obviamente, la técnica de datos de panel presenta ventajas e inconvenientes (Balatagi, 2010; Wooldridge, 2010; Greene, 2011). En este trabajo nos centramos en los modelos más conocidos de datos de panel: modelos de efectos fijos y modelos de efectos aleatorios.

Así, con el modelo de efectos fijos la especificación sería la siguiente:

$$
\begin{aligned}
& \text {SALUD }_{i}=\beta_{0}+\beta_{1} Y_{i t}+\beta_{2} \text { OFERTA }_{i t}+\beta_{3} \text { SS }_{i t}+\beta_{4} D E S C_{i t}+i \alpha_{i}+\varepsilon_{i}, \\
& \text { GSPC }_{i}=\beta_{0}+\beta_{1} Y_{i}+\beta_{2} \text { DEMANDA }_{i}+\beta_{3} \text { OFERTA }_{i}+\beta_{4} S S_{i t}+\beta_{5} \text { DESC }_{i t}+i \alpha_{i}+\varepsilon_{i},
\end{aligned}
$$

donde se considera que existe un término constante y diferente para cada individuo, y se supone que los efectos individuales son independientes entre sí.

Por otra parte, el modelo de efectos aleatorios viene dado por:

$$
\begin{aligned}
& S A L U D_{i t}=\beta_{0}+\beta_{1} Y_{i t}+\beta_{2} \text { OFERTA }_{i t}+\beta_{3} S_{i t}+\beta_{4} \text { DESC }_{i t}+\alpha+u_{i}+\varepsilon_{i t}, \\
& \text { GSPC }_{i t}=\beta_{0}+\beta_{1} Y_{i}+\beta_{2} \text { DEMANDA }_{i}+\beta_{3} \text { OFERTA }_{i}+\beta_{4} S_{i t}+\beta_{5} \text { DESC }_{i t}+\alpha+u_{i}+\varepsilon_{i t},
\end{aligned}
$$

donde se considera que los efectos individuales no son independientes entre sí.

De esta manera, la heterogeneidad no observable en el modelo de efectos fijos se incorpora en la ordenada al origen del modelo y con el modelo de efectos aleatorios, se incorpora en el término de error. Por último en el proceso de estimación econométrica se ha realizado el contraste de Hausman (1978) para veri-

sanitario subcentral respecto al total de gasto sanitario. La principal fuente de datos fiscales es la Government Finance Statistics (GFS) del Fondo Monetario Internacional (FMI). La salvedad en este caso es que los indicadores de descentralización fiscal deben ser calculados netos de transferencias intergubernamentales. Por otro lado, para el caso de la descentralización de ingresos también se utilizaría un indicador de tipo fiscal a partir de la misma fuente anterior: la ratio de ingresos subcentrales como fuente de financiación sanitaria respecto al total de ingresos destinados a sanidad.

Si bien estas estadísticas recopilan información para más de 100 países, el ejercicio de comparar país por país del grado de descentralización sanitaria es limitada. El motivo es que existe una doble contabilidad de las transferencias intergubernamentales en las estimaciones del FMI de gasto sanitario. Así, las transferencias sanitarias de niveles superiores de gobierno son consideradas tanto como gasto de estos niveles donantes como gasto de los gobiernos receptores. El problema en este caso aparece cuando estas transferencias son de nivelación hacia los gobiernos subcentrales. Así, para construir en este caso estos indicadores de descentralización sanitaria desde la perspectiva del gasto hemos tenido en cuenta que computar las transferencias sanitarias como gasto de los niveles superiores de gobierno puede infraestimar el nivel real de autonomía subcentral en su gestión sanitaria. Mientras, el nivel de autonomía subcentral en ingresos puede ser sobreestimado. Esto se debe a que la Government Finance Statistics clasifica a los impuestos en compartidos, recargos e impuestos en que el tipo y/o la base son decididos por el gobierno central como fuente de ingresos propios a nivel subcentral. Para obtener los indicadores empleados en este estudio se ha tenido también en cuenta que la OECD en un estudio de 1999 ha hecho grandes esfuerzos en mejorar la información disponible sobre ingresos clasificando los impuestos según la autonomía fiscal de cada gobierno encargado de ellos. 
ficar si el modelo que mejor se ajusta a los datos es el de efectos fijos o el de efectos aleatorios.

Teniendo en cuenta las anteriores puntualizaciones, los modelos planteados contrastan la relación de los resultados en salud, así como de los gastos sanitarios per cápita de los países, con diferentes variables explicativas para el caso europeo. Así, en la Tabla 1 se describen las principales variables utilizadas en el estudio así como sus correspondientes definiciones en función de que recojan factores demográficos, socioeconómicos, estados de salud y estilos de vida así como recursos, tipos y grado de descentralización de los sistemas sanitarios. La Tabla 2 recoge los principales estadísticos descriptivos dónde algunas de las variables se expresan en términos logarítmicos a fin de tener una mejor comprensión de las mismas posteriormente al estimar como elasticidades.

Tabla 1

Variables y definiciones. Unión Europea

\begin{tabular}{|c|c|c|}
\hline \multicolumn{2}{|c|}{ VARIABLES DEPENDIENTES } & DEFINICIÓN \\
\hline \multicolumn{2}{|l|}{ SALUD: EVN, MORTIF } & $\begin{array}{l}\text { Esperanza de vida al nacer (años). Mortalidad infantil } \\
\text { (muertes por cada } 1000 \text { nacidos vivos). Fuente: OECD } \\
\text { Health Data }\end{array}$ \\
\hline \multicolumn{2}{|l|}{ GSPC } & $\begin{array}{l}\text { Gasto Sanitario (per capita US\$ PPP). Fuente: OECD Health } \\
\text { Data }\end{array}$ \\
\hline \multicolumn{3}{|c|}{ VARIABLES INDEPENDIENTES } \\
\hline VECTOR & VARIABLES & DEFINICIÓN \\
\hline $\begin{array}{l}\text { Factores } \\
\text { Demográficos }(E D A D)\end{array}$ & $\begin{array}{l}\text { ENV } \\
\text { (F.DEMANDA) }\end{array}$ & $\begin{array}{l}\text { Envejecimiento (\% población mayor de } 65 \text { años sobre el } \\
\text { total). Fuente: OECD Health Data }\end{array}$ \\
\hline \multirow{2}{*}{$\begin{array}{l}\text { Factores } \\
\text { Socioeconómicos } \\
\text { (SOCE) }\end{array}$} & Y & $\begin{array}{l}\text { Producto Nacional Bruto (per cápita US\$ PPP). Fuente: } \\
\text { OECD Health Data }\end{array}$ \\
\hline & $\begin{array}{l}\text { DESEMPLEO } \\
\text { (F.DEMANDA) }\end{array}$ & $\begin{array}{l}\text { Porcentaje de población en desempleo sobre el total. } \\
\text { Fuente: OECD Health Data }\end{array}$ \\
\hline $\begin{array}{l}\text { Estado de Salud y } \\
\text { estilos de vida } \\
\text { (SALUD) }\end{array}$ & $\begin{array}{l}\text { MORTINF } \\
\text { (F.DEMANDA) }\end{array}$ & $\begin{array}{l}\text { Mortalidad Infantil (muertes por } 1000 \text { nacidos vivos). Fuente: } \\
\text { OECD Health Data }\end{array}$ \\
\hline \multirow{3}{*}{$\begin{array}{l}\text { Recursos sanitarios } \\
\text { (OFERTA) }\end{array}$} & CAMAS (F.OFERTA) & $\begin{array}{l}\text { Número de Camas por } 1000 \text { habitantes. Fuente: OECD } \\
\text { Health Data }\end{array}$ \\
\hline & $\begin{array}{l}\text { MEDICOS } \\
\text { (F.OFERTA) }\end{array}$ & $\begin{array}{l}\text { Número de Médicos de Medicina General (densidad por } \\
1000 \text { habitantes). Fuente: OECD Health Data }\end{array}$ \\
\hline & $\begin{array}{l}\text { ESTANCIAS } \\
\text { (F.OFERTA) }\end{array}$ & Estancia media (días). Fuente: OECD Health Data \\
\hline $\begin{array}{l}\text { Tipo de Sistema } \\
\text { Sanitario (SISTEMA) }\end{array}$ & SS & $\begin{array}{l}1 \text { si el país tiene un Sistema Sanitario de Seguridad Social, } 0 \\
\text { si el país tiene un Sistema Nacional de Salud }\end{array}$ \\
\hline \multirow{2}{*}{$\begin{array}{l}\text { Grado de } \\
\text { descentralización } \\
\text { sanitaria } \\
(D E S C E N T R)\end{array}$} & DESCINGR & $\begin{array}{l}\text { Ratio de ingresos sanitarios subcentrales respecto al total de } \\
\text { ingresos destinados a sanidad. Fuente: Government Finance } \\
\text { Statistics (GFS) del Fondo Monetario internacional (FMI) }\end{array}$ \\
\hline & DESCGASTO & $\begin{array}{l}\text { Ratio de gasto sanitario subcentral respecto al total de gasto } \\
\text { sanitario. Fuente: Government Finance Statistics (GFS) del } \\
\text { Fondo Monetario internacional (FMI) }\end{array}$ \\
\hline
\end{tabular}

Fuente: Elaboración propia. 
Tabla 2

Estadísticos descriptivos

\begin{tabular}{|l|c|c|c|c|c|}
\hline \multicolumn{1}{|c|}{ Variable } & Media & Desv. Estándar & Min & Max & Observ. \\
\hline EVN & 15.6606 & 1.9579 & 10.9000 & 20.6000 & 315 \\
\hline MORTIF & 5.0089 & 1.6150 & 1.8000 & 11.0000 & 315 \\
\hline $\log$ GSPC & 7.6500 & 0.3918 & 6.5073 & 8.4735 & 315 \\
\hline $\log Y$ & 10.1370 & 0.3633 & 9.2775 & 11.3970 & 315 \\
\hline DESEMPLEO & 3.5597 & 1.5785 & 0.6000 & 9.9000 & 315 \\
\hline CAMAS & 4.1203 & 1.5949 & 1.9000 & 10.1000 & 315 \\
\hline MÉDICOS & 3.1100 & 0.6586 & 1.6000 & 4.6800 & 315 \\
\hline ESTANCIAS & 10.2319 & 5.8801 & 3.4000 & 34.1000 & 314 \\
\hline DESCINGR & 14.7144 & 9.9295 & 5.1800 & 37.7200 & 315 \\
\hline DESCGASTO & 23.6155 & 13.2972 & 8.2400 & 59.8400 & 315 \\
\hline SS & 0.4000 & 0.4907 & 0.0000 & 1.0000 & 315 \\
\hline
\end{tabular}

Fuente: Elaboración propia a partir de la Tabla 1.

\section{RESULTADOS}

Los resultados de las estimaciones se presentan de manera sintética resumidos en las Tablas 3-6. Así, en las dos primeras se analizan los determinantes de los resultados en salud y su relación con la descentralización fiscal; mientras en las últimas se presentan los determinantes del gasto sanitario y los efectos de la descentralización en el mismo. En ambos objetivos, se toma de manera separada la influencia de factores de demanda, de oferta o de ambos conjuntamente, teniendo en cuenta en la última columna de cada tabla el impacto del grado de descentralización sanitaria de cada país.

La estimación de dichos modelos muestra una alta bondad del ajuste en términos de los estadísticos y los coeficientes que son significativos y tienen en la mayoría de los casos los signos esperados de acuerdo con el criterio económico a priori y estadístico. Es decir, los coeficientes estimados resultan altamente significativos tanto individualmente (contraste de la $t$ de Student) como en conjunto (contraste de F). El $\mathrm{R}^{2}$ cuadrado ajustado presenta un valor alto.

\subsection{Descentralización y Salud}

Tal y como era de esperar, la renta tiene un claro efecto negativo sobre un indicador de salud (mortalidad infantil, Tabla 3) mientras que su influencia es positiva para el otro (la esperanza de vida al nacer, Tabla 4). Por otro lado, el número de médicos de medicina general por cada 1000 habitantes en el país resulta ser la única variable de oferta explicativa con efecto claramente positivo en ganancias de esperanza de vida, en la Tabla 4 (al contrario que el número relativo de camas). En cuanto a las variables de descentralización tanto de gasto como de ingreso, resultan de igual forma ser significativas, con signo contrario según la variable dependiente de la que se trate en cada caso. 
Tabla 3

Descentralización y Salud.

Variable dependiente: Mortalidad Infantil (MORTIF). UE.

\begin{tabular}{|c|c|c|c|c|c|}
\hline $\begin{array}{c}\text { Variables } \\
\text { explicativas }\end{array}$ & $\log Y$ & OFERTA & $\begin{array}{l}\text { TIPO DE } \\
\text { SISTEMA }\end{array}$ & \multicolumn{2}{|c|}{ DESCENTRALIZACIÓN } \\
\hline \multirow[t]{2}{*}{$\log Y$} & $-4.4777 \quad$ *** & -3.9900 & -4.4140 & -4.5398 & $* \star \star *$ \\
\hline & $(-29.91)$ & $(-17.19)$ & $(-16.32)$ & $(-17.79)$ & \\
\hline \multirow[t]{2}{*}{ CAMAS } & & 0.1571 & 0.0970 & 0.0054 & \\
\hline & & $(2.38)$ & $(1.25)$ & $(0.07)$ & \\
\hline \multirow[t]{2}{*}{ MÉDICOS } & & -0.2335 & -0.0557 & -0.07214 & \\
\hline & & $(-1.57)$ & $(-0.32)$ & $(-0.44)$ & \\
\hline \multirow[t]{2}{*}{ ESTANCIAS } & & -0.0077 & -0.0183 & -0.0203 & \\
\hline & & $(-0.59)$ & $(-1.32)$ & $(-1.57)$ & \\
\hline \multirow[t]{2}{*}{ DESCINGR } & & & & -0.2007 & 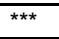 \\
\hline & & & & $(-6.71)$ & \\
\hline \multirow[t]{2}{*}{ DESCGASTO } & & & & 0.0889 & 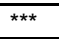 \\
\hline & & & & (3.01) & \\
\hline R-cuadrado & 0.5113 & 0.5770 & 0.5336 & 0.6084 & \\
\hline $\begin{array}{c}\text { F/ Wald Statistic and } \\
\text { Prob(F) }\end{array}$ & $\begin{array}{l}894.66 \\
(0.0000)\end{array}$ & $\begin{array}{l}864.19 \\
(0.0000)\end{array}$ & $\begin{array}{l}222.85 \\
(0.0000)\end{array}$ & $\begin{array}{c}179.18 \\
(0.0000)\end{array}$ & \\
\hline $\begin{array}{l}\text { Hausman Statistic and } \\
\text { Prob(Hausman) }\end{array}$ & -38.05 & $\begin{array}{c}7.87 \\
(0.0963)\end{array}$ & $\begin{array}{c}71.97 \\
(0.0000)\end{array}$ & $\begin{array}{c}27.95 \\
(0.0001)\end{array}$ & \\
\hline
\end{tabular}

Nota: ${ }^{* *},{ }^{* *} y *$ denota significatividad estadística a los niveles del 1, 5 y $10 \%$ respectivamente. $t$ valores entre paréntesis.

Fuente: Elaboración propia.

\section{Tabla 4}

Descentralización y resultados en Salud.

Variable dependiente: Esperanza de vida al nacer (ENV). UE.

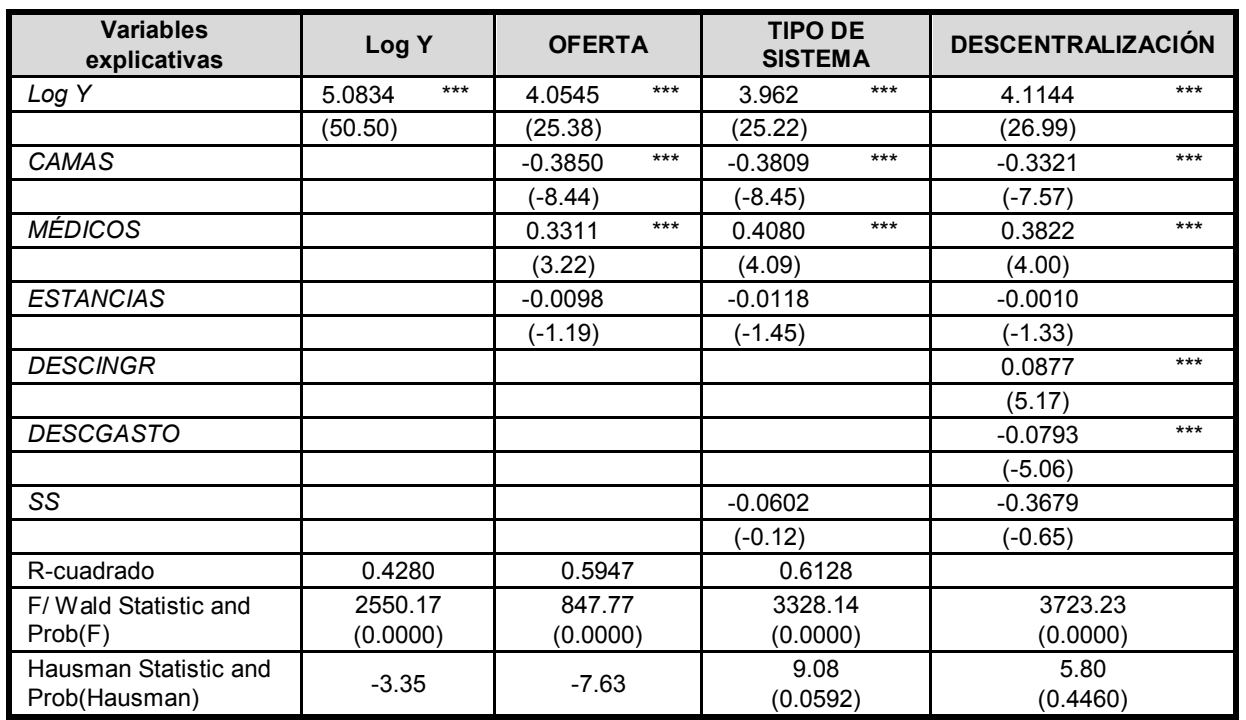

Nota: ${ }^{* * *},{ }^{* *} y{ }^{*}$ denota significatividad estadística a los niveles del 1, 5 y $10 \%$ respectivamente. $t$ valores entre paréntesis.

Fuente: Elaboración propia. 


\subsection{Descentralización y Gasto}

En este caso, tal y como muestra la Tabla 5, nos encontramos con que es la variable renta la que tiene un claro efecto positivo sobre los gastos sanitarios de los principales países europeos, con además elasticidades cercanas a la unidad en el caso de considerarla tanto por separado como en el caso de incluir conjuntamente factores de demanda y de oferta. Por otro lado, en los países europeos un mayor grado de envejecimiento estaría presionando claramente sobre los gastos sanitarios. En cuanto a las variables de oferta, tanto un mayor número relativo de camas como de médicos, serían indicativas de mayores costes en términos de gastos sanitarios (dándose el clásico problema que aparece en economía de la salud de la demanda inducida por la oferta) no detectándose entonces la presencia de economías de escala. Referente asimismo a la descentralización, observamos un efecto positivo sobre el gasto si la descentralización se produce básicamente vía gastos, mientras que el signo inverso presenta la descentralización por el lado de los ingresos.

\section{Tabla 5}

Descentralización y Gasto.

Variable dependiente: log gasto sanitario per cápita (GSPC). UE.

\begin{tabular}{|c|c|c|c|c|c|c|}
\hline \multirow{2}{*}{$\begin{array}{c}\begin{array}{c}\text { Variables } \\
\text { explicativas }\end{array} \\
\log Y\end{array}$} & $\log Y$ & OFERTA & DEMANDA & $\begin{array}{l}\text { OFERTA Y } \\
\text { DEMANDA }\end{array}$ & \multicolumn{2}{|c|}{ DESCENTRALIZACIÓN } \\
\hline & $1.1610^{* * *}$ & $1.1432^{* \star *}$ & $0.9566 \quad$ *** & $0.9076 \quad$ *** & 0.9170 & *** \\
\hline & $(74.79)$ & $(41.16)$ & $(28.19)$ & $(21.94)$ & $(21.10)$ & \\
\hline \multirow[t]{2}{*}{$E N V$} & & & 0.0105 & 0.0168 & 0.0207 & *** \\
\hline & & & $(1.84)$ & $(2.86)$ & $(3.29)$ & \\
\hline \multirow[t]{2}{*}{ MORTINF } & & & -0.0034 & -0.0350 & -0.0336 & *** \\
\hline & & & $(-5.18)$ & $(-5.31)$ & $(-4.94)$ & \\
\hline \multirow[t]{2}{*}{ DESEMPLEO } & & & $-0.0170 \quad * \star \star$ & -0.0183 & -0.0153 & 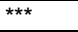 \\
\hline & & & $(-3.66)$ & $(-3.96)$ & $(-3.24)$ & \\
\hline \multirow[t]{2}{*}{ CAMAS } & & $0.0214^{\star \star \star *}$ & & 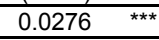 & 0.0276 & $\star \star \star *$ \\
\hline & & $(2.70)$ & & $(3.60)$ & $(3.44)$ & \\
\hline \multirow[t]{2}{*}{ MÉDICOS } & & $0.0407 \quad$ ** & & $0.0480^{* \star *}$ & 0.0388 & ** \\
\hline & & $(2.28)$ & & $(3.04)$ & $(2.37)$ & \\
\hline \multirow[t]{2}{*}{ ESTANCIAS } & & $\begin{array}{r}- \\
0.0019\end{array}$ & & $-0.0034 \quad$ ** & -0.0030 & *** \\
\hline & & $(-1.36)$ & & $(-2.54)$ & $(-2.25)$ & \\
\hline \multirow[t]{2}{*}{ DESCINGR } & & & & & -0.0067 & ** \\
\hline & & & & & $(-2.04)$ & \\
\hline \multirow[t]{2}{*}{ DESCGASTO } & & & & & 0.0097 & $\star \star \star$ \\
\hline & & & & & (3.16) & \\
\hline \multirow[t]{2}{*}{ SS } & & & & 0.0103 & & \\
\hline & & & & $(0.15)$ & & \\
\hline R-cuadrado & 0.8238 & 0.8475 & 0.8623 & 0.8874 & 0.8837 & \\
\hline $\begin{array}{l}\text { F/ Wald Statistic and } \\
\text { Prob(F) }\end{array}$ & $\begin{array}{l}5593.34 \\
(0.0000) \\
\end{array}$ & $\begin{array}{l}1431.87 \\
(0.0000) \\
\end{array}$ & $\begin{array}{l}1612.80 \\
(0.0000) \\
\end{array}$ & $\begin{array}{l}6844.54 \\
(0.0000) \\
\end{array}$ & $\begin{array}{l}781.90 \\
(0.0000) \\
\end{array}$ & \\
\hline $\begin{array}{l}\text { Hausman Statistic } \\
\text { and Prob(Hausman) }\end{array}$ & -6.07 & $\begin{array}{c}11.47 \\
0.0218\end{array}$ & $\begin{array}{l}42.51 \\
0.0000\end{array}$ & $\begin{array}{c}7.42 \\
0.3861\end{array}$ & -4.67 & \\
\hline
\end{tabular}

Nota: ${ }^{* *},{ }^{* *} y{ }^{*}$ denota significatividad estadística a los niveles del 1, 5 y $10 \%$ respectivamente. $t$ valores entre paréntesis.

Fuente: Elaboración propia. 
De igual forma, y con objeto de extender el análisis, se propone introducir en el modelo el retardo de nuestra variable dependiente, es decir, convertimos nuestro panel en dinámico al introducir un retardo del gasto sanitario per cápita a fin de considerar el efecto "anclaje" que pueda tener en el nivel del gasto el del año anterior.

Utilizando el estimador de Arellano y Bover (1995) y Blundell and Bond (1998), para realizar las distintas estimaciones obtenemos la Tabla 6 de resultados en la que volvemos a diferenciar especificaciones que tengan en cuenta no sólo al logaritmo de la renta sino también a factores de oferta, demanda, de ambas o los propios relativos a la descentralización, tanto de gasto como de ingreso.

\section{Tabla 6}

Descentralización y Gasto.

Variable dependiente: log gasto sanitario per cápita (GSPC). UE. Panel Dinámico

\begin{tabular}{|c|c|c|c|c|c|c|}
\hline \multirow{2}{*}{$\begin{array}{c}\begin{array}{c}\text { Variables } \\
\text { explicativas }\end{array} \\
\log Y\end{array}$} & $\log Y$ & OFERTA & DEMANDA & $\begin{array}{l}\text { OFERTA Y } \\
\text { DEMANDA }\end{array}$ & \multicolumn{2}{|c|}{ DESCENTRALIZACIÓN } \\
\hline & $0.2245^{\star \star \star *}$ & $0.1857^{\star \star \star *}$ & $0.1975^{* \star *}$ & 0.1698 & 0.1624 & *** \\
\hline & $(4.09)$ & $(3.61)$ & $(4.43)$ & $(4.66)$ & $(4.56)$ & \\
\hline \multirow[t]{2}{*}{ GSPC - 1} & $0.8009^{\star \star *}$ & $0.8090 \quad$ *** & $0.7686 \quad$ *** & 0.7731 & 0.7717 & *** \\
\hline & $(20.67)$ & $(23.31)$ & $(30.20)$ & $(29.75)$ & $(31.06)$ & \\
\hline \multirow[t]{2}{*}{ ENV } & & & 0.0093 & 0.0078 & 0.0085 & \\
\hline & & & $(1.52)$ & $(1.25)$ & $(1.56)$ & \\
\hline \multirow[t]{2}{*}{ MORTINF } & & & -0.0050 & -0.0054 & -0.0061 & ${ }^{*}$ \\
\hline & & & $(-1.59)$ & $(-1.65)$ & $(-1.88)$ & \\
\hline \multirow[t]{2}{*}{ DESEMPLEO } & & & $-0.0130 \quad * * *$ & $-0.0137 \quad * * *$ & -0.0138 & *** \\
\hline & & & $(-4.56)$ & $(-5.66)$ & $(-5.24)$ & \\
\hline \multirow[t]{2}{*}{ CAMAS } & & -0.0111 & & -0.0023 & -0.0020 & \\
\hline & & $(-1.34)$ & & $(-0.30)$ & $(-0.29)$ & \\
\hline \multirow[t]{2}{*}{ MÉDICOS } & & 0.0102 & & 0.0118 & 0.0128 & \\
\hline & & $(0.90)$ & & $(1.34)$ & $(1.46)$ & \\
\hline \multirow[t]{2}{*}{ ESTANCIAS } & & -0.0002 & & -0.0012 & -0.0013 & ** \\
\hline & & $(-0.49)$ & & $(-2.08)$ & $(-2.26)$ & \\
\hline \multirow[t]{2}{*}{ DESCINGR } & & & & & -0.0014 & \\
\hline & & & & & $(-0.84)$ & \\
\hline \multirow[t]{2}{*}{ DESCGASTO } & & & & & 0.0019 & \\
\hline & & & & & $(1.34)$ & \\
\hline \multirow[t]{2}{*}{ SS } & & & & -0.0158 & -0.0002 & \\
\hline & & & & $(-0.56)$ & $(-0.00)$ & \\
\hline $\begin{array}{l}\text { Arellano-Bond } \\
\text { test for } A R(1) \text { in } \\
\text { first differences } \\
\text { and Prob(F) }\end{array}$ & $\begin{array}{l}-2.1469 \\
(0.0318)\end{array}$ & $\begin{array}{c}-2.1954 \\
(0.0281)\end{array}$ & $\begin{array}{l}-2.2741 \\
(0.0230)\end{array}$ & $\begin{array}{c}-2.3204 \\
0.0203\end{array}$ & $\begin{array}{c}-2.3465 \\
0.0190\end{array}$ & \\
\hline $\begin{array}{l}\text { Arellano-Bond } \\
\text { test for } A R(1) \text { in } \\
\text { first differences } \\
\text { and Prob(F) }\end{array}$ & $\begin{array}{c}-0.3775 \\
0.7058\end{array}$ & $\begin{array}{r}-0.47217 \\
(0.6368)\end{array}$ & $\begin{array}{c}0.0023 \\
(0.9982)\end{array}$ & $\begin{array}{c}-0.0717 \\
0.9428\end{array}$ & $\begin{array}{c}-0.0541 \\
0.9568\end{array}$ & \\
\hline
\end{tabular}

Nota: ${ }^{* * *},{ }^{* *} y{ }^{*}$ denota significatividad estadística a los niveles del 1, 5 y 10\% respectivamente. $t$ valores entre paréntesis.

Fuente: Elaboración propia. 
Como comentario general, y en comparativa con los resultados anteriormente mostrados, merece la pena finalmente subrayar el hecho de que las únicas variables significativas resultan ser la renta y el gasto retardado en un período, el desempleo, la tasa de mortalidad infantil y una variable de utilización sanitaria (las estancias). Así, el gasto de un ejercicio vendría explicado entorno a un $80 \%$ por el gasto del periodo anterior (justificación de la hipótesis de "anclaje" del gasto sanitario e indicativa, de la dificultad de variar significativamente el nivel de gasto sanitario de un período a otro). Y es que la renta también mantiene aquí su impacto positivo sobre el gasto, pero con elasticidades que en ningún caso llegan a superar la unidad como para suponer que la sanidad se comportase como un bien de lujo.

\section{CONCLUSIONES}

En este trabajo se han analizado los principales determinantes de los resultados en salud y gasto en sanidad, y se ha propuesto un modelo explicativo de su comportamiento, para el caso de diferentes países europeos en un contexto descentralizado. Los resultados muestran que el factor más importante en la explicación, tanto del volumen de gasto público sanitario como de los resultados en salud, es la renta. La mayor mortalidad infantil y la mayor tasa de desempleo tendrían su correspondencia en unos menores gastos sanitarios. Por su parte, el envejecimiento, las variables de oferta así como un mayor grado de descentralización desde la perspectiva del gasto tendrían el efecto inverso. En el caso de los resultados en salud, se observa de nuevo un efecto de la descentralización inverso para gastos-ingresos. Y un efecto, principalmente significativo para las variables de oferta.

En relación con otros estudios publicados, es importante señalar que en los últimos años, multitud de estudios [Mahal et al. (2000); Robalino et al., (2001); Habibi et al. (2003); Cantarero y Pascual (2008); Jiménez-Rubio et al. (2010) o Jiménez-Rubio (2011)], han analizado el impacto de descentralización en diversos indicadores objetivos de salud de la población, tales como mortalidad infantil y esperanza de vida al nacer. El consenso encontrado revela que existe un efecto positivo de la descentralización, mas como apuntan Jiménez-Rubio et al. (2010) éste sería limitado. De igual forma, existe una amplia literatura en la economía de la salud centrada en el análisis de los determinantes del gasto sanitario (en este sentido Costa-Font y Moscone (2008), analizan los determinantes del gasto público regional en un contexto de descentralización y de relaciones interterritoriales para el caso español), si bien la mayor parte de la misma se ha centrado en el análisis de la renta como principal factor (en concreto, en el debate en la Economía de la Salud acerca de si la elasticidad renta del gasto sanitario es mayor o menor que 1), la literatura apunta a que el grado de descentralización del sistema de salud en cada país puede ser de importancia 
al relacionarse con el nivel final de gasto sanitario, siendo más relevante en el caso de los gastos que en el de los ingresos dado el carácter asimétrico de los procesos de descentralización (Leu, 1986; Jiménez y Smith, 2005; Cantarero, 2006). Así, nuestro trabajo sería una nueva contribución sobre los determinantes de los resultados en salud y gasto sanitario, considerando la descentralización fiscal como variable clave, si bien, ésta tendría finalmente un efecto ambiguo.

No obstante esto, pensamos que se precisa analizar con mayor profundidad los posibles efectos no lineales de las variables, así como modelizar el impacto del cambio tecnológico sobre el gasto sanitario (como variaciones en el stock de conocimiento relativo a los tratamientos asistenciales). Eso no significa que conociésemos su influencia a priori pues si las nuevas tecnologías sanitarias consiguiesen abaratar el coste de los tratamientos podrían reducirse los costes sanitarios asociados a dicho cambio tecnológico (Navarro y Hernández, 2006). En cambio, si apareciesen mayores gastos relativos a los nuevos tratamientos, entonces dicho cambio tecnológico estaría asociado con mayores gastos sanitarios y la orientación de políticas públicas debería tener en cuenta eso por lo que nuestra propuesta consistiría en evaluar mejor la inversión en nuevas tecnologías sanitarias dentro del gasto sanitario y su aportación en términos de salud más que centrarse de manera principal como hasta ahora en ajustar el gasto en el capítulo de personal lo que además de modificar la oferta puede desincentivar en muchos casos a los profesionales sanitarios.

En definitiva, la conclusión principal de nuestro estudio es que la renta es el factor más importante en la explicación del volumen de gasto público sanitario $\mathrm{y}$ de los resultados en salud. Existe pues un efecto inverso para la descentralización fiscal de ingresos-gastos, siendo más favorable por el lado de los ingresos.

\section{REFERENCIAS BIBLIOGRÁFICAS}

ARELLANO, M.; BOVER, O. (1995). "Another look at the instrumental variable estimation of error-components models" en Journal of Econometrics, 68(1), pp.29-51.

ATELLA, V.; MARINI; G. (2004a). "Is health care expenditure really a luxury good? Reassessment and new evidence based on OECD data". Working Paper, http://papers.ssrn.com/abstract=383004.

ATELLA, V.; MARINI, G. (2004b). "The determinants of health care expenditure: a never ending story?”. Working Paper, http://papers.ssrn.com/abstract=607162.

BAC, C.; LE PEN, Y. (2002). "An international comparison of health expenditure determinants". 10th International Conferences on Panel Data, Berlin, July 5-6.

BALTAGI, B.H. (2010) Econometric analysis of panel data. $4^{\mathrm{a}}$ ed. Chichester: Wiley, 351p.

BANTING, K.G.; CORBETT, S. (2002). Health Policy and Federalism. A Comparative perspective on multi-level governance. McGill-Queen's University Press, Kingston. 
BARROS, P.P. (1998). "The black-box of health care expenditure growth determinants". Health Economics, 7, pp. 533-544.

BLAZQUEZ, C., GONZALEZ, N., MORENO, P. (2013). "Pharmaceutical expenditure as a determinant of health outcomes in EU countries". Estudios de Economía Aplicada, 31-2, pp. 379-396

BLUNDELL R.; BOND S. (1998). "Initial conditions and moment restrictions in dynamic panel data models", Journal of Econometrics, 87(1), pp.115-43.

BROWN, M.C. (1987). "Caring for profit: economic dimensions of Canada's health industry". Fraser Institute, Vancouver.

CABASES-HITA, J.M. (2006). La financiación del gasto sanitario desde la perspectiva de la experiencia comparada. Bilbao: Fundación BBVA; 2006. 400p.

CABASES-HITA, J.M. (2010). La financiación del gasto sanitario en España. Valoración del sistema de financiación, medida de la necesidad relativa y equidad. Fundación BBVA; 2010. Informe Economía y Sociedad.

CANTARERO, D. (2006). "Determinantes del gasto sanitario: un análisis empírico para el caso europeo", Estudios de Economía Española, 228, FEDEA, Madrid.

CANTARERO, D.; PASCUAL, M. (2008). "Analysing the impact of fiscal decentralization on health outcomes: empirical evidence from Spain", Applied Economic Letters, (15), pp.109-111.

COSTA-FONT, J.; MOSCONE, F. (2008). "The impact of decentralization and interterritorial interactions on Spanish health expenditure", Empirical Economics, 34(1), pp.167-184.

COSTA-FONT, J. (2012). "Fiscal Federalism and European Health System Decentralization: A Perspective", LEQS, Paper No. 55/2012.

DI MATTEO, L. (2000). "The determinants of the public-private mix in Canadian health care expenditures: 1975-1996". Health Policy, 52, pp. 87-112.

DI MATTEO, L. (2003). "The income elasticity of health care spending: a comparison of parametric and non-parametric approaches". European Journal of Health Economics, 4, pp. 20-29.

GBESEMETE, K.P.; GERDTHAM, U.-G. (1992). "Determinants of Health Care Expenditure in Africa: A Cross-Sectional Study". Word Development, 20, pp. 303-308.

GETZEN, T.E. (2001). "Aggregation and the measurement of health care costs". Manuscript.

GERDTHAM, U.G.; JONSSON, B.; MACFARLAN, M.; OXLEY, H. (1998). The determinants of health expenditure in the OECD countries. En ZWEIFEL, P. (ed.): Health, The Medical Profesión and Regulation, Kluwer Academic Publishers, Dordrecht.

GERDTHAM, U.G.; JONSSON, B. (2000). "International comparisons of health expenditure: theory, data and econometric analysis". En CULYER, A.J, NEWHOUSE, J.P. (eds.): Handbook of health economics, Vol. 1, Elsevier, Amsterdam.

GREENE WH. (2011). Econometric Analysis. $7^{\text {a }}$ ed. New Jersey: Pearson Education.

HABIBI, N.; HUANG, C.; MIRANDA, D.; MURILLO, V.; RANIS, G.; SARKAR, M. (2003). "Decentralization and Human Development in Argentina", Journal of Human Development, 4(1).

HENDERSON, J.W. (2002). Health economics and policy. South-Western, 2nd edn, Thomson Learning, Cincinnati. 
HAUSMAN, J.A. (1978). "Specification Tests in Econometrics" en Econometrica, 46, pp. 1013-1029.

JIMÉNEZ-RUBIO, D.; CANTARERO-PRIETO, D.; PASCUAL-SÁEZ, M. (2010). "Is fiscal decentralization good for you health? Evidence from a panel OECD countries". XVIII Encuentro de Economía Pública 2011.

JIMÉNEZ-RUBIO, D. (2011). "The impact of decentralization of health services on health outcomes: evidence from Canada". Applied Economics, 43(26), pp. 3907-3917.

JIMENEZ, D.; SMITH, P. (2005). "Decentralisation of health care and its impact on health outcomes". Discussion Papers in Economics, №. 2005/10, University of York.

KANAVOS, P.; MOSSIALOS, E. (1996). "The methodology of international comparisons of health care expenditures: any lessons for health policy?" LSE Health Discussion Paper, No. 3, London.

KLEIMAN, E. (1974). The determinants of national outlay on Health. Macmillan, London.

LEU, R.E. (1986). "The public-private mix and international health care costs". En CULYER, A.J., JONSSON, B. (Eds.): Public and Private health services, Basil Blackwell, Oxford, p. 41-63.

MAHAL, A.; SRIVASTAVA, V.; SANAN, D. (2000). "Decentralization and its impact on public service provision on health and education sectors: the case of India", En: Dethier J (Ed.), Governance, Decentralization and Reform in China, India and Russia, Kluwer Academic Publishers and ZEF, London, p.77.

NAVARRO, J.L., HERNANDEZ, E. (2006). "Health technologies assessment: analysing the role of uncertainty". Estudios de Economía Aplicada, 24-3, pp. 731-754.

NEWHOUSE, J.P. (1977). Medical care expenditure: a cross-national survey. Journal of Human Resources, 12, pp. 115-125.

OECD: OECD Health Data File.

PARKIN, D.; MCGUIRE, A.; YULE, B. (1987). "Aggregate health care expenditures and national income". Journal of Health Economics, 6, pp. 109-127.

REINHARDT, U.E.; HUSSEY, P.S.; ANDERSON, G.F. (2002). "Cross National Comparisons of Health Care Systems using OECD data 1999". Health Affairs, May/June, pp. 169-182.

ROBALINO, D. A.; PICAZO, O. F.; VOETBERG, A. (2001). "Does fiscal decentralization improve health outcomes? Evidence from a cross-country analysis", Washington DC: World Bank, Policy Research Working Paper, 2565.

SEN, A. (2005): "Is health care a luxury? New evidence from OECD Data". International Journal of Health Care Finance and Economics, 5, pp. 147-164.

URBANOS, R. (2004). "Tendencias internacionales en la financiación del gasto sanitario". Papeles de Trabajo del Instituto de Estudios Fiscales, 19.

WENDT, C. (2009). "Mapping European healthcare systems: a comparative analysis of financing, service provision and access to healthcare", Journal of European Social Policy, 19, pp. 432-445.

WOOLDRIDGE, J.M. (2010). Econometric analysis of cross section and panel data. $2^{\mathrm{a}}$ ed. London: The MIT Press, $1064 \mathrm{p}$. 


\section{ANEXO}

Resultados en salud y gasto y PNB
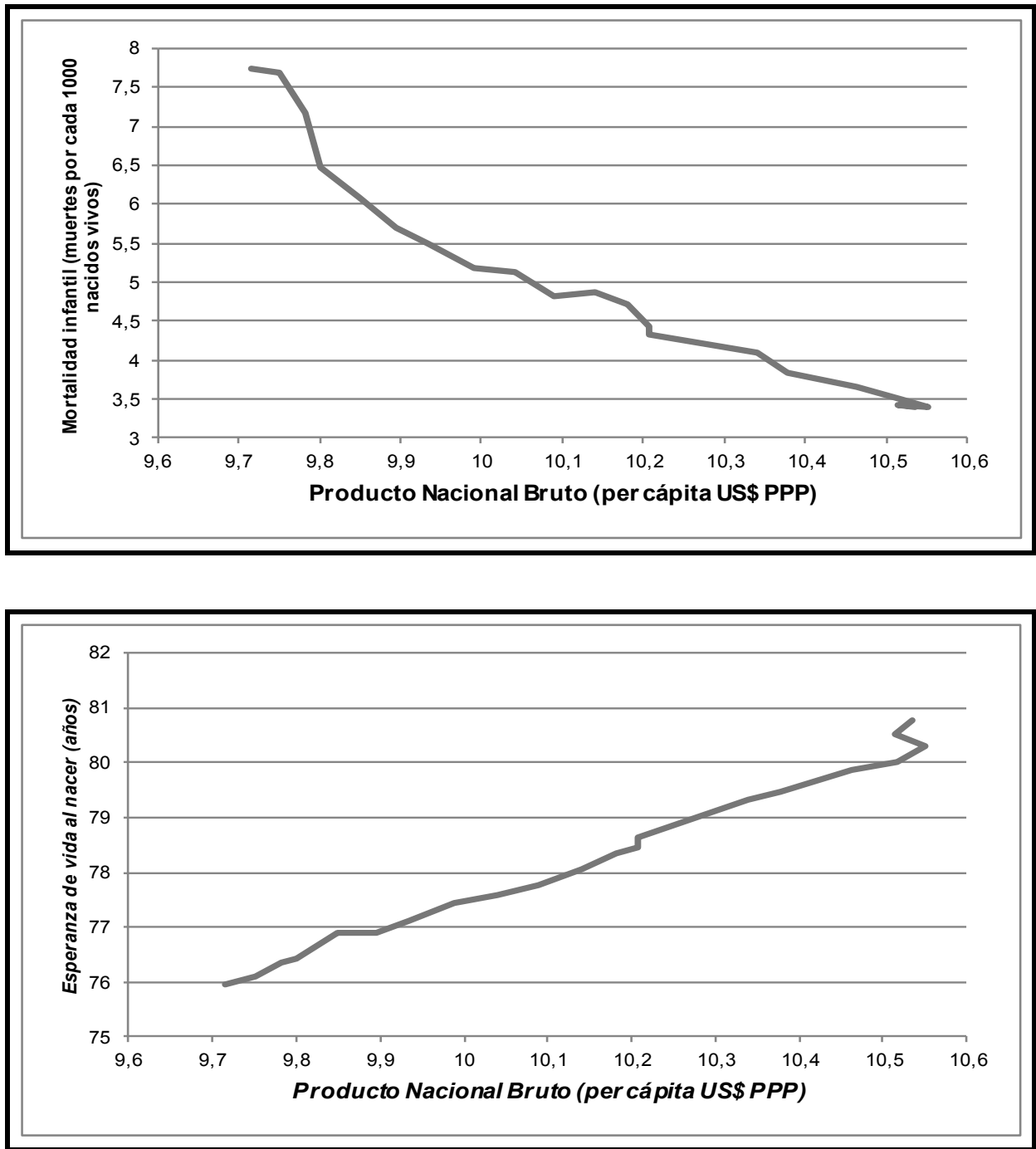


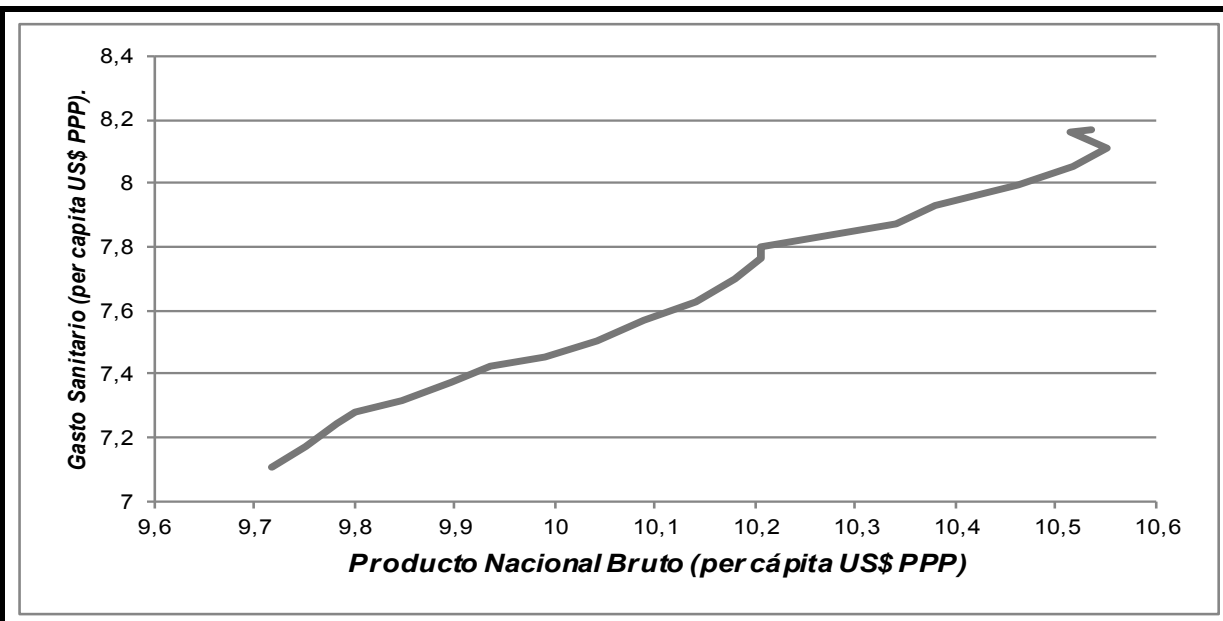

Fuente: Elaboración propia a partir de OECD Health Data. 
